\title{
A comparative Study of Reassigned Conventional Wavelet Transform for Machinery Faults Detection
}

\author{
Ahmed M.Abdelrhman ${ }^{1, a^{*}}$, M. Salman Leong ${ }^{2, b}$, Lim Meng Hee $^{3, \mathrm{c}}$ \\ and Wai Keng Ngui ${ }^{4, d}$
}

\author{
${ }^{1}$ Department of Mechanical Engineering, Faculty of Engineering and Technical Studies, University \\ of Kordofan, Sudan \\ ${ }^{2,4}$ Institute of Noise and Vibration, Universiti Teknologi Malaysia, Kuala Lumpur, Malaysia \\ ${ }^{3}$ RAZAK School of Engineering \& Advanced Technology, Universiti Teknologi Malaysia, Malaysia \\ aahmedrabak@yahoo.com, bsalman@ic.utm.my, cmhlim@ic.utm.my, waikeng84@yahoo.com
}

Keywords: Morlet wavelet, Feature extraction, Fault detection.

\begin{abstract}
Application of Fast Fourier Transform (FFT) in machinery faults detection is known to be only effective if fault is of repetitive in nature and considering severe. While minor and transient faults are usually remain undetected based on vibration spectrum analysis. Wavelet analysis is relatively new technique which is still suffered from inadequately in its time-frequency resolution. In this paper, ahmedrabak_time wavelet is proposed based on the wavelet reassignment technique for Morlet mother wavelet. The proposed wavelet analysis is compared to the conventional wavelet analysis for machinery faults detection based on simulated signal. The results showed that the proposed wavelet has a better resolution than conventional wavelet analysis which could clearly indicate the presence and the location of the fault.
\end{abstract}

\section{Introduction}

Machinery fault detection usually involves the analysis of vibration signal to estimate the current health condition of the machines. Minor and transient faults in machinery such as gear teeth notch, minor blade and bearing faults could generate non-stationary signals (transient and impact), whereby their occurrence will either change abruptly or disappear in time or could not generate sufficient energy to be noticeable in the vibration spectrum. These small defects if not detected early could propagate over time and potentially compromise the total integrity of the machine [1]. For these faults, time information is crucial in identifying these events for effective machinery condition monitoring and diagnosis. The conventional Fast Fourier Transform analysis is deemed less effective in detecting these types of signals. In contrast, Wavelet analysis methods are useful in capturing, identifying, and analyzing local, multi-scale, and non-stationary processes, thus enabling the analysis of trends, breakdown points, discontinuities, and self-similarity [2]. Nevertheless, Wavelet analysis has its own pitfalls as well. One noticeable pitfall is its lack of sufficient resolution in both time and frequency domain. In other words, it is difficult to analyze signals with multiple frequency components which are completely overlapped in the wavelet maps [3, 4]. Frequency interference and spectral smearing in the results of wavelet maps can lead to difficulties in the interpretation of results and thus sometimes can cause misdetections of machinery faults as well [5]. In this case, improving the time and frequency resolution of the wavelet is deemed important to enhance fault detection capability of wavelet analysis. In this paper, a new wavelet analysis is proposed based on the reassignment technique of wavelet analysis to improve the wavelet time resolution and thus to achieve a better concentration of signals energy distribution in time domain.

\section{Wavelet Analysis Transform}

Conventional FFT technique analyses signals with a constant window for all frequencies. In comparison, wavelet transforms analyses signals in a more flexible way by using different window 
functions for each associated frequency in the signal. As a result, it allows wavelet analysis to have high signals localization in the time-frequency plane by changing the mother wavelet shapes to become narrow at high frequencies and wider at lower frequencies. For machinery fault diagnosis, Continuous Wavelet Transform (CWT) is often used because it can provide detailed information for the signal at different levels of resolution and also can determine the similarity of the signal to the daughter mother wavelet function [6]. The basic configuration of CWT produces high frequency resolution at low frequencies region; and high time resolution at high frequencies region. As such, results always suffer from poor resolution and interpretation of results.

\section{Morlet Mother Wavelet}

The optimum mother Wavelet selection is a major consideration and the main challenge in the application of the wavelet transform [7]. In mechanical fault diagnosis, Morlet wavelet is one of the most popular mother wavelet to be used. It is because that Morlet wavelet has higher similarity in its characteristics to vibration signals in comparison to many other mother wavelet functions [8]. Morlet wavelet transform is defined in Eq. 1 as:

$$
\psi(t)=\exp \left(-\frac{\beta^{2} t^{2}}{2}\right) \cos (\pi t)
$$

The bandwidth parameter $(\beta)$ could be adjusted to adapt to the signals with any decaying rate. Morlet wavelet time-frequency resolution could also be adapted to various type of signals in dilation with scale $a$ and translation with $b$ [9]. The son of Morlet wavelet is described in Eq. 2 as:

$$
\psi_{a, b}(t)=\exp \left[-\frac{\beta^{2}(t-b)^{2}}{a^{2}}\right] \cos \left[-\frac{\pi(t-b)}{a}\right]
$$

Here, the translation parameter $b$ represents the inspected signal. Parameter $\beta$ balances the timefrequency resolutions of Morlet wavelet and controls the shape of the mother wavelet. For instance, when $\beta$ increased, the time resolution will increase. Therefore, the best time resolution of Morlet wavelet can be achieved when $\beta$ approaching to infinity $[6,9]$. For each signal, there is always an optimal value of $\beta$ exists to achieve the best time and frequency resolutions. One more important parameter of wavelet analysis is the center of frequency $F_{c} . F_{c}$ is an important parameter to estimate the amplitudes of the wavelet coefficients.

Addison, et al [10] commented that, in practice, central frequency of mother wavelet analysis less than 5 is more oscillatory and thus allows for better resolution of wavelet. As the main objective of his study is to improve the time resolution of the wavelet analysis, a new mother wavelet is formulated based on the reassignment technique of Morlet wavelet. The formulated wavelet was named as ahmedrabak_time, and was added to the wavelet toolbox in Matlab software. The added mother wavelet is a family of type 4 which have no associated scaling function therefore it is only be applied for continuous wavelet analysis. A wider effective support ([-8 8]) is chosen for the proposed wavelet to provide more accurate results. 


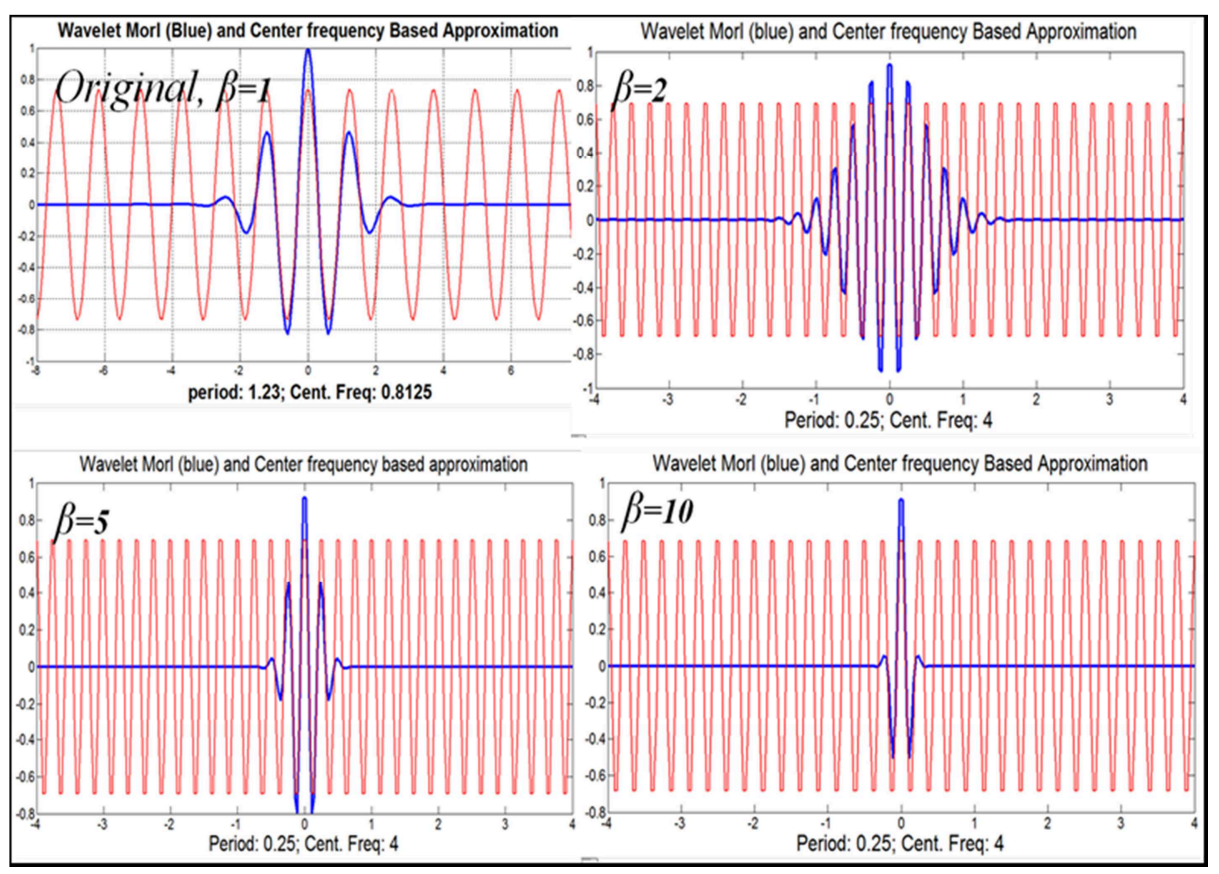

Fig. 1 Morlet wavelet shapes at different center frequency and $\beta$ values.

Fig. 1 shows the Morlet wavelet shapes at centre frequency of 0.8125 and 4 , and at four different values of $\beta(1,2,5$ and 10). The optimal wavelet parameter for best time resolution is achieved when $\beta=10$ and centre of frequency $=4$.

\section{Signal simulation study}

The effectiveness of the newly devised wavelets was tested using simulated signals and its results were compared to the conventional wavelet analysis. A composite signal consists of $160 \mathrm{~Hz}$, $220 \mathrm{~Hz}$, and $260 \mathrm{~Hz}$ with additive noise was generated using MATLAB at sampling frequency of 1024 and time duration of 0.05 second. This signal could represent one cycle data of a rotor system with three stages of blades each with 8,11 , and 13 numbers of blades running at $1200 \mathrm{RPM}$. The amplitude of first stage blade pass frequency (BPF) was set to be higher to represent the condition of faulty blade at stage 1 . The simulated signal was then analysed using both Morlet wavelet and the newly added ahmedrabak_time wavelets. Fig. 2 shows the results of the signal simulation study. 

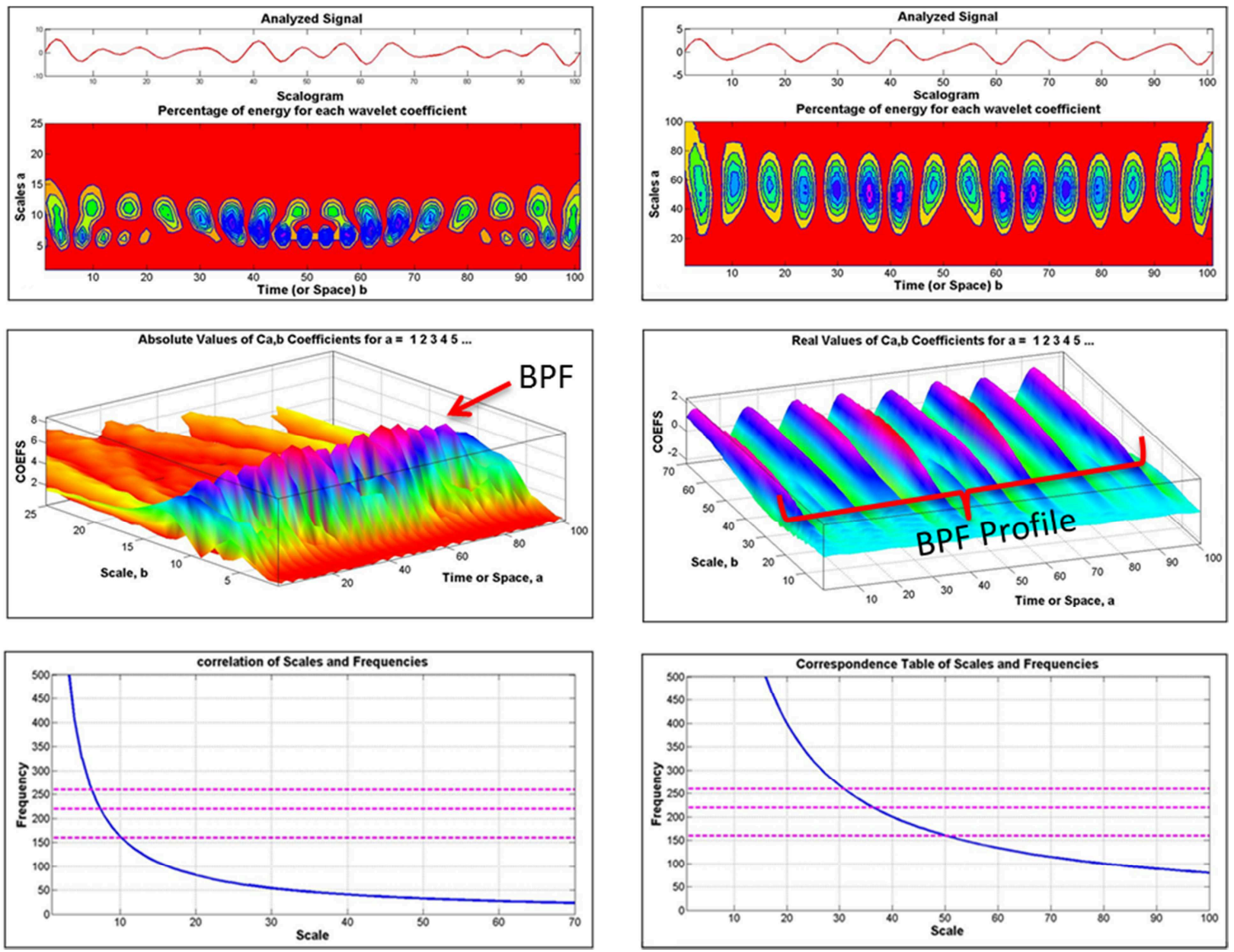

(a) Morlet wavelet analysis results

(b) Ahmedrabak_time wavelet analysis results

Fig. 2 Compression of the absolute values of Morlet with real value of proposed wavelet

Fig. 2 compared the absolute values of Morlet with real value of proposed wavelet. Analysis results of Morlet wavelet as shown in Fig. 2a provides evidence that the effects of lack of resolution due to frequency overlapping and interference. This can be seen as the three BPFs were lumped together and overlapped into each other, making the analysis result difficult to be interpreted. On the other hand, analysis results ahmedrabak_time wavelet as shown in Fig. 2b demonstrated that the proposed method is more effective in representing more detailed time information of the signal as compared to Morlet wavelet results. Severe intense indications at the BPF region of the faulty stage could be clearly seen in the wavelet map. BPF profiles of faulty blade stage can be seen clearly as compared to Morlet wavelet analysis. In this instance, the number and the position of the faulty blade stage could be reasonably estimated. In addition, the newly added wavelet also has demonstrated a shorter processing time as ( 0.25 second $)$ compared to Morlet wavelet (1.23 second).

\section{Conclusion}

A wavelet with higher time resolution is presented in this study. The proposed wavelet provided a real wavelet coefficient values and provided more detailed results to analyses features in signal, based on visual study as compared to the conventional Morlet wavelet analysis. The proposed wavelet also allows the detection of blade faults and the identification of the faulty blades stage in rotor system. It is believed that, the proposed method could be used for other condition monitoring applications such as those for gears and bearings faults diagnosis purposes. 


\section{References}

[1] B. Yang, "Blade containment evaluation of civil aircraft engines," Chinese Journal of Aeronautics, vol. 26, pp. 9-16, 2// 2013.

[2] M. Misiti, Y. Misiti, G. Oppenheim, and J. Poggi, "Wavelet toolbox," 1996.

[3] Ahmed M.Abdelrhman, M. S. Leong, L. M. Hee, and W. K. Ngui, "Application of Wavelet Analysis in Blade Faults Diagnosis for Multi-Stages Rotor System," Applied Mechanics and Materials, 2013.

[4] Ahmed M. Abdelrhman, M. S. Leong, L. M. Hee, and K. H. Hui, "Vibration Analysis of Multi Stages Rotor for Blade Faults Diagnosis," Advanced Materials Research, vol. 845, pp. 133-137, 2014.

[5] P. W. Tse, W.-x. Yang, and H. Tam, "Machine fault diagnosis through an effective exact wavelet analysis," Journal of Sound and Vibration, vol. 277, pp. 1005-1024, 2004.

[6] D. Zhang and W. Sui, "The optimal morlet wavelet and its application on mechanical fault detection," in Wireless Communications, Networking and Mobile Computing, 2009. WiCom'09. 5th International Conference on, 2009, pp. 1-4.

[7] W. K. Ngui, M. S. Leong, Ahmed.M.Abdelrhman, and L. M. Hee, "Wavelet Analysis: Mother Wavelet Selection Methods," Applied Mechanics and Materials, 2013.

[8] J. Rafiee, M. Rafiee, and P. Tse, "Application of mother wavelet functions for automatic gear and bearing fault diagnosis," Expert Systems with Applications, vol. 37, pp. 4568-4579, 2010.

[9] J. Lin and L. Qu, "Feature Extraction Based On Morlet Wavelet And Its Application For Mechanical Fault Diagnosis," Journal of Sound and Vibration, vol. 234, pp. 135-148, 2000.

[10]P. Addison, J. Watson, and T. FENG, "Low-oscillation complex wavelets," Journal of Sound and Vibration, vol. 254, pp. 733-762, 2002. 